\title{
Analysis of the CW-mode optically controlled microwave switch
}

\author{
Sangil Lee and Yasuo Kuga \\ Department of Electrical Engineering, University of Washington
}

\begin{abstract}
Optical-microwave interaction has been emphasized in optically reconfigurable antenna arrays (ORA) due to the unique advantage that transparency between the optical control signals and microwave signals makes the antenna less susceptible to jamming. One of the important parts in ORA is an optically controlled microwave switch (OMS) as synaptic elements. A gap-structure OMS has been developed as a low-cost and simple device which operates in all frequencies. However, the OMS is essentially operated in a CW-mode, and adverse effects are observed in the CWmode operation. Although a CW-mode OMS has been investigated previously, a detailed analysis has not been reported. In this paper, we present an analysis, and the numerical simulations are compared with the previous measurements.
\end{abstract}

Keywords: Microwave switch, optical control, CW-mode laser, coplanar waveguide (CPW)

\section{INTRODUCTION}

Optical control has been employed to control the microwave signals mainly because of the fast response, immunity from EMI, high power handling, good isolation between controlling and controlled devices, and possibilities for monolithic integration with other devices ${ }^{1}$. Since the reporting of picosecond photoconductivity, various microwave devices using the photoconductivity effect have been developed. One simple application for the photoconductivity effect is the direct excitation of a gap on a semiconductor transmission line with a laser light. The transmission characteristics can be controlled with the generated free carriers; the "ON" and "OFF" state can be used for a switching device or an attenuator. Since Jayaraman and Lee reported the first microwave device using picosecond photoconductivity in 1972, various optically controlled microwave devices have been presented. In 1975, Auston demonstrated the optically controlled microwave switching and gating technique based on a silicon-based microstrip line from the voltage pulses ${ }^{2}$. However, the long carrier lifetime of a silicon wafer limits the switching speed, thus the materials having a shorter carrier lifetime, such as GaAs were suggested for ultra-fast optoelectronics. In 1977, Lee presented the picosecondswitching device with GaAs, and Defonzo reported the picosecond photoconductivity in germanium films in 1981. Besides the switching and gating devices, many other applications have been presented, which include sampling, picosecond active pulse shaping, waveform generation, microwave modulation, generation of microwave bursts, optical detection, phase shifters, and attenuator.

In recent years vigorous research on an optically reconfigurable antenna array has been carried out due to the unique advantage that there is no interaction between the optical control signals and microwave antenna signals, which makes the antenna less susceptible to jamming ${ }^{3}$. Since the original concept for the optically reconfigurable antenna (ORA) was proposed by Dempsey and Bevensee in 1989, several different applications have been presented ${ }^{4}$. One simple structure for an ORA is a set of tunable monopole antenna segments that are connected by optically controlled microwave switches, as synaptic elements ${ }^{5}$. Depending on the "ON" and "OFF" state of the OMS, the length of the monopole antenna changes, and the operating frequency can be controlled. Another application for the ORA is an optically reconfigurable scanning antenna (ORSA) proposed by the Hughes Co. Each dipole antenna in the printed dipole arrays is designed for short dipoles, therefore, the length of the dipole is much shorter than the wavelength of the transmitting signal. If the OMSs between dipoles are activated with laser light and the dipoles are connected to adjacent dipoles, the effective lengths of the dipoles are increased. Thus, the antenna radiation pattern can be controlled changing

leesi@ee.washington.edu; phone 1206 543-2159; fax 1206 543-3842; Dept. of Electrical Engineering, Univ. of Washington, Box 352500, Seattle, WA 98195-2500

ykuga@u.washington.edu; phone 1206 543-0478; fax 1206 543-3842 
the different length of the dipoles with the OMSs. This concept can be used optically reconfigurable scanning antenna. One of the most important parts of the ORA and ORSA are the optically controlled microwave switches as synaptic nodes. The photovoltaically-biased field effect transistor (PV-FET) can be adapted for optically controlled microwave switches $^{6}$. The concept has been successfully developed at low RF frequencies up to $10 \mathrm{GHz}$. However, the PV-FET may have limitations at higher frequencies. The photoconductive gap can be used as an optically controlled microwave switch for a lower-cost structure and a wider operating frequency range. The basic idea of the gap-structure OMS is the photoconductivity effect of the gap on a silicon-based transmission line ${ }^{7-9}$. When a laser light is applied on a gap, free carriers are generated that can be used for the conducting channel. Hence, the "ON" and "OFF" mechanism is easily produced. If the switch is used only for the very short "ON" time duration, which is shorter than the recombination rate of the generated free carriers, high photoconductivity can be easily obtained with the relatively low incident optical power. However, optically controlled microwave switches for ORAs are essentially operated in CW or quasi-CW mode. Unfortunately, in the $\mathrm{CW}$ or quasi-CW mode operation, many adverse effects have been observed. The photoconductivity is significantly decreased due to the carrier recombination and the carrier diffusion, and the resulting low carrier density makes it difficult to obtain a good insertion loss. To increase the photoconductivity, we may need to limit the substrate so as to have a longer carrier lifetime, however, the longer carrier lifetime may limit the switching speed. Also, the longer diffusion length due to the longer carrier lifetime may change the dielectric substrate to a lossy media with generated carriers. In this case, the insertion loss cannot be improved even though the carrier density is increased. Another possible way to increase the photoconductivity is exciting a gap with a higher optical power, however increasing the power is not recommended. Furthermore, the very high incident power may cause other problems, such as carrier burning. In this paper, a detailed analysis for the insertion loss of gap-structure OMS in CW-mode operation is given with emphasis on the transmission line structure.

\section{MATERIAL PREPARATION}

In picosecond photoconductivity a direct bandgap material such as GaAs can be effectively used as a substrate material for a fast switching device. However, in the $\mathrm{CW}$-mode operation the shorter carrier lifetime of the direct bandgap materials may decrease the number of generated free carriers due to fast carrier recombination; thus, it may not be possible to obtain good insertion loss or attenuation with direct bandgap materials. A substrate material suitable for optimal operation in CW-mode applications must be considered. It is known that a silicon wafer provides many advantages as a substrate material for solid-state microwave devices: low cost, mature technology, good thermal conductance, and possibilities for monolithic integration with other devices or circuits. Above all, the low-loss characteristic of the silicon wafer may be one of the most important features for microwave and millimeter wave devices. Any semiconductor material can be used for the substrate of the optically controlled microwave devices. However, there are two important factors to be considered as a proper substrate material: the number of generated free carriers produced with the photoconductivity effect, and a low loss property.

A photoconductivity effect is one of the basic phenomena that occur in semiconductor materials. When laser light is applied on a semiconductor substrate with higher energy than the bandgap of the substrate material, free carriers (both electron and holes) are generated. The semiconductor substrate becomes lossy and the photoconductivity is increased $^{10}$. The photoconductivity with light excitation is given by

$$
\sigma=\sigma_{0}+e \Delta n\left(\mu_{n}+\mu_{p}\right)
$$

where $\sigma_{0}$, is dark photoconductivity, $e$ is electronic charge, and $\mu_{n}$ and $\mu_{p}$ are the mobilities for electrons and holes, respectively $\Delta n=\Delta p$, since electrons and holes are always created in pairs. This equation shows the relation between the photoconductivity and the generated free carriers, and we clearly see that the photoconductivity is directly proportional to the generated free carriers. Thus, we may be able to determine the important factors for obtaining higher photoconductivity through describing $\Delta n$, which is an optical property of the solid-state material and given by,

$$
\Delta n=\frac{W_{p} / A}{h \omega} \alpha(1-R) S
$$

where $W_{p}$ is optical pulse energy (optical peak power * pulse width), $A$ is excited area, $\alpha$ is the absorption coefficient, 
$h \omega$ is the photon energy to excite electrons, $R$ is the surface reflectivity, and $S$ is the relative spectral response of the semiconductor material exhibiting a peak response at $\lambda_{0}$. Although this equation does not include surface recombination and carrier diffusion, it may present very important facts for choosing a substrate material to obtain higher photoconductivity with the same optical input power. A semiconductor material that has a higher absorption coefficient and longer carrier lifetime generates a higher concentration of free carriers with the same optical input power. The absorption coefficient is a function of the wavelengths of incident light. A higher absorption coefficient is provided with a shorter wavelength of the applied light. Although a silicon wafer has an approximately ten times lower absorption coefficient than that of GaAs at a wavelength of $632 \mathrm{~nm}$, the carrier lifetime of direct bandgap materials are generally more than one thousand times shorter than that of indirect bandgap materials. As a consequence, it may be very difficult to obtain a higher concentration of generated free carriers with direct bandgap materials in CW-mode.

Another important requirement for a good substrate for solid-state microwave devices is a low-loss dielectric property. The dielectric property is determined by the resistivity of the substrate material. If the resistivity of the substrate material is too low in the dark state, the substrate is already a lossy medium. In this case, the substrate material may not be adequate for the transmission of the microwave signals. A high-resistivity silicon wafer is seen to be the best choice due to its very long carrier lifetime and good dielectric properties. There are two kinds of silicon wafers depending on the crystal growth process: one is a regular silicon wafer with Czochralski (CZ) process and the other is a float-zone wafer with zone process. Only zone processes can grow a highly purified silicon wafer, and a high purity silicon wafer leads to very long carrier lifetime and very high resistivity. Therefore, high-resistivity silicon wafers with the float-zone process have been chosen for the CW-mode optically controlled microwave devices.

\section{TRANSMISSION LINES FOR THE GAP-STRUCTURE OMS}

Transmission lines such as microstrip lines, strip lines, coplanar strips, and CPWs are commonly used for the interconnections of the integrated circuit. A microstrip line is one of the most popular transmission lines mainly due to the quasi-TEM mode propagation, which allows an easy analysis with wide-band operation. Another advantage of microstrip lines is an easy integration with microwave circuits and simple connections with coaxial cables. Therefore, microstrip lines have been actively used as the transmission line of optically controlled microwave switches. However, the microstrip line can be used only for very low-duty photoconductivity or picosecond photoconductivity due to carrier diffusion. Most electric fields are distributed in the plasma region between the signal line (top layer) and the ground plane (bottom layer). If a very low-duty laser light is applied, the plasma depth is just few $\mu \mathrm{m}$ from the surface of a gap. Thus, the plasma layer can be effectively used for recovering the gap and then most microwave signals can be transmitted without a significant loss. However, in CW-mode operation we need to limit the substrate material to having a long carrier lifetime to generate more carriers. If carrier lifetime is increased, the diffusion lengths of carriers are also increased. Fig. 1 shows the carrier distribution (a) if the carrier diffusion length is the same or longer than the substrate thickness and (b) if the carrier diffusion length is shorter than the substrate thickness. The diffusion length becomes approximately $500 \mu \mathrm{m}$ with $1 \times 10^{-4} \mathrm{sec}$ of carrier lifetime, which is the same as or longer than the substrate thickness. In this case, the optically generated free carriers are used for making the substrate lossy rather than for the conducting channel of a gap. Fig. 2 and 3 show the HFSS (High Frequency Structure Simulator) simulation results of the insertion loss depending on the effective photoconductivity for the microstrip line. The insertion loss is improved up to $2000 \mathrm{~S} / \mathrm{m}$ of the effective photoconductivity, but the insertion loss is increased with higher than $2000 \mathrm{~S} / \mathrm{m}$ as shown in Fig. 2. As a consequence, the microwave signals cannot be transmitted through the plasma due to the generated free carriers.

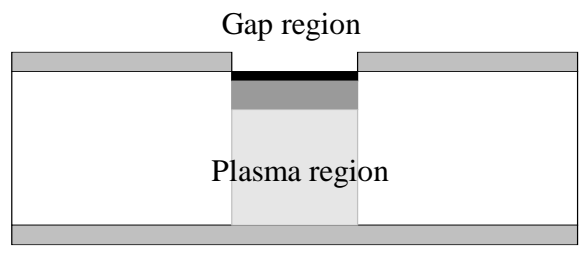

(a)

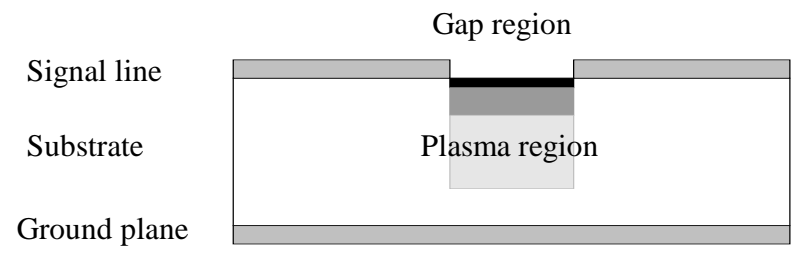

(b)

Fig. 1. The generated carrier distribution for the microstrip line structure: (a) carrier diffusion length is longer than the substrate thickness (b) carrier diffusion length is shorter than the substrate thickness. 


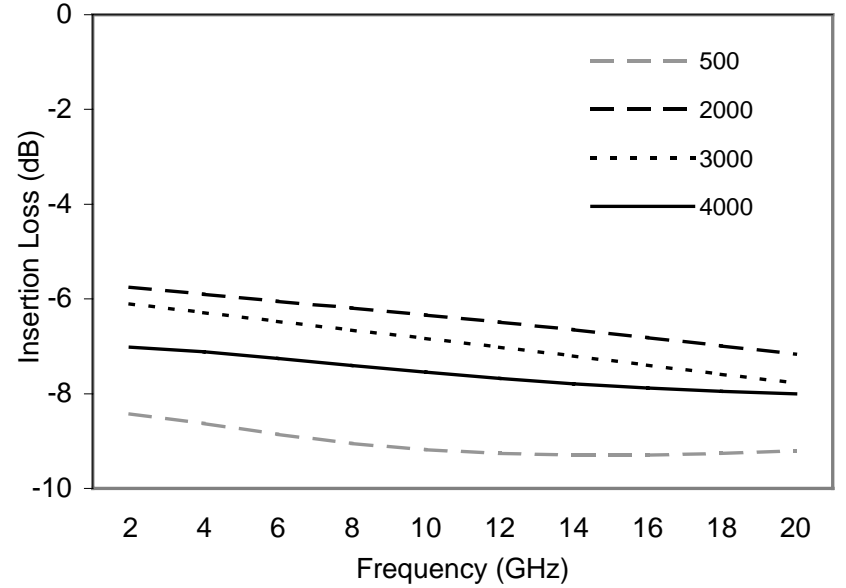

(a)

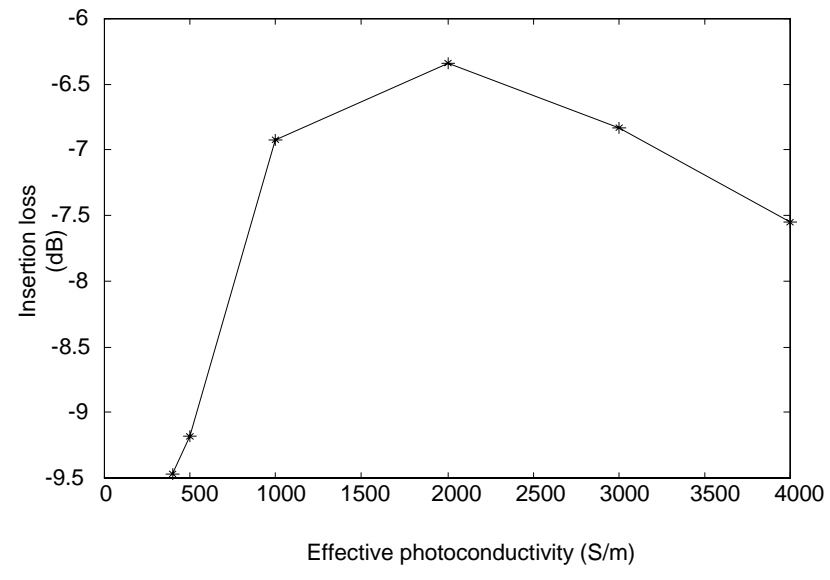

(b)

Fig. 2. HFSS simulation for the insertion loss of the microstrip line structure (carrier diffusion length is longer than the substrate thickness).

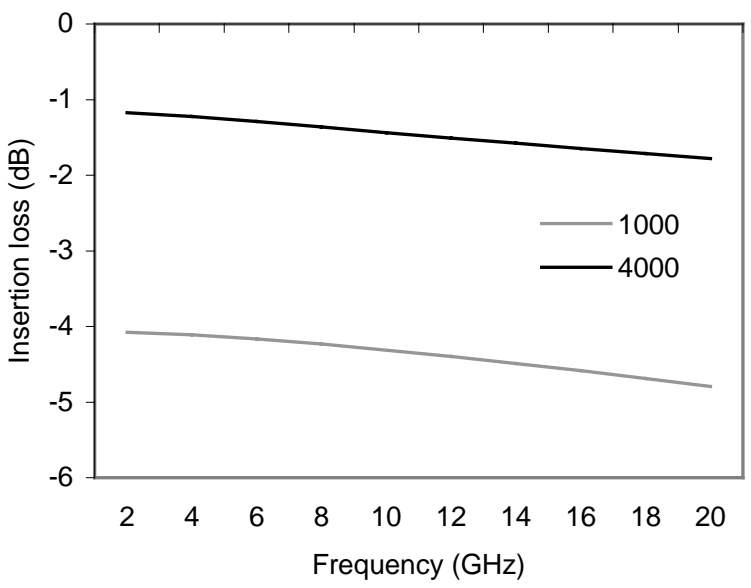

Fig. 3. HFSS simulation for the insertion loss of the microstrip line structure (carrier diffusion length is shorter than the substrate thickness).
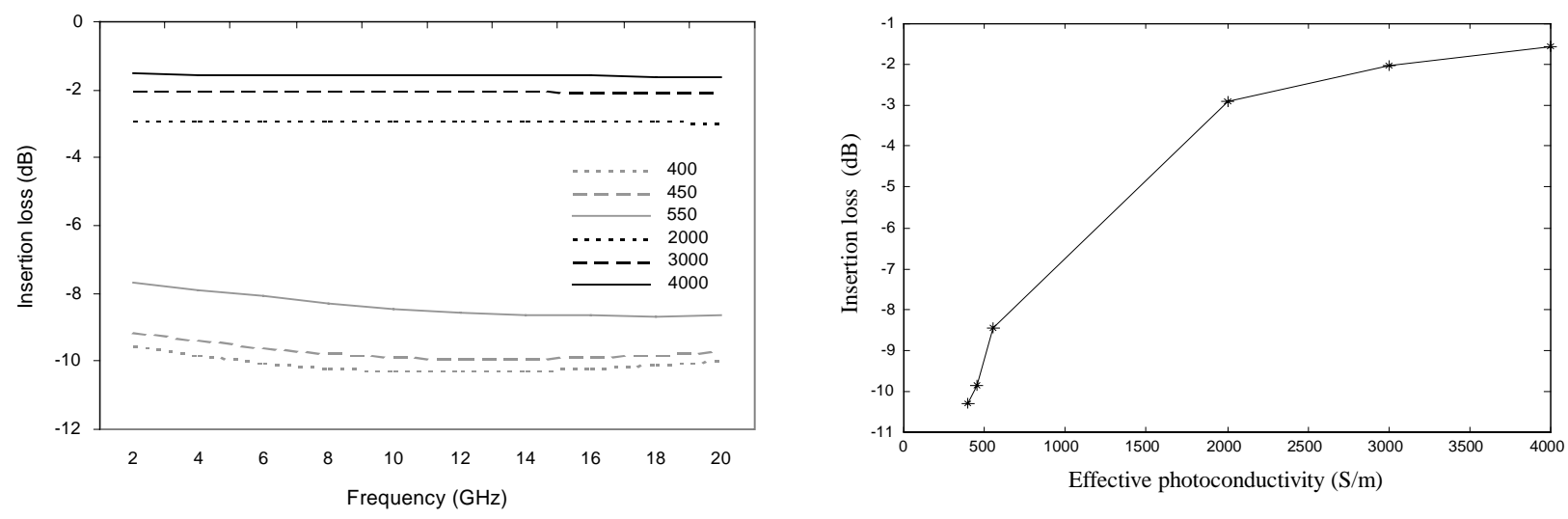

Fig. 4. HFSS simulation for the insertion loss of the CPW structure: (a) as a function of frequency at difference photoconductivity (b) as a function of photoconductivity at $10 \mathrm{GHz}$. 
If a microstrip line is required for a CW-mode OMS, only a substrate having a short carrier life or a very thick substrate is acceptable. As shown in Fig. 3, if the plasma depth is shallower than the substrate thickness, the insertion loss can be significantly improved with higher photoconductivity. As a result, a microstrip line can be used for the OMS with picosecond photoconductivity, but it may not be a proper structure for a CW-mode OMS, or it can be used only under limited conditions.

Another commonly used transmission line in microwave integrated circuits is a CPW. The term "coplanar lines" is used for those transmission lines in which all the conductors are in the same plane. A distinct advantage of the coplanar waveguide is in the fact that mounting of lumped (active or passive) components in shunt or series configuration is much easier and drilling of holes or slots through the substrate is not needed. One of the most important features of the CPW in the OMS is the electric field distribution. Most of the electric field is distributed in between the signal line and ground plane on the same top surface. Thus, the propagation loss may not be significant if the optically generated free carriers are confined under the signal line. Fig. 4 shows the HFSS simulation results for the insertion loss of the CW-mode OMS on a CPW. We may improve the insertion loss in proportion to photoconductivity with the CPW structure.

\section{INSERTION LOSS: SIMULATIONS AND EXPERIMENTS}

The transmission characteristics of the CW-mode OMS on a CPW are simulated using a finite element code HFSS. The model used for the simulation is based on the effective photoconductivity and the effective plasma depth of the gap region ${ }^{11-13}$. As shown in Fig. 6, the simulation models are specified for both with and without considering the carrier diffusion. First, we need to calculate the effective photoconductivity and the effective plasma depth. From the calculated photoconductivity and plasma depth, the insertion loss can be obtained with the HFSS simulations. On the contrary, we may inversely obtain the effective photoconductivity from the measured insertion loss. Thus, the inversely obtained photoconductivity is compared with the calculated values. As a result, we are able to see the differences of the insertion loss due to the carrier diffusion.

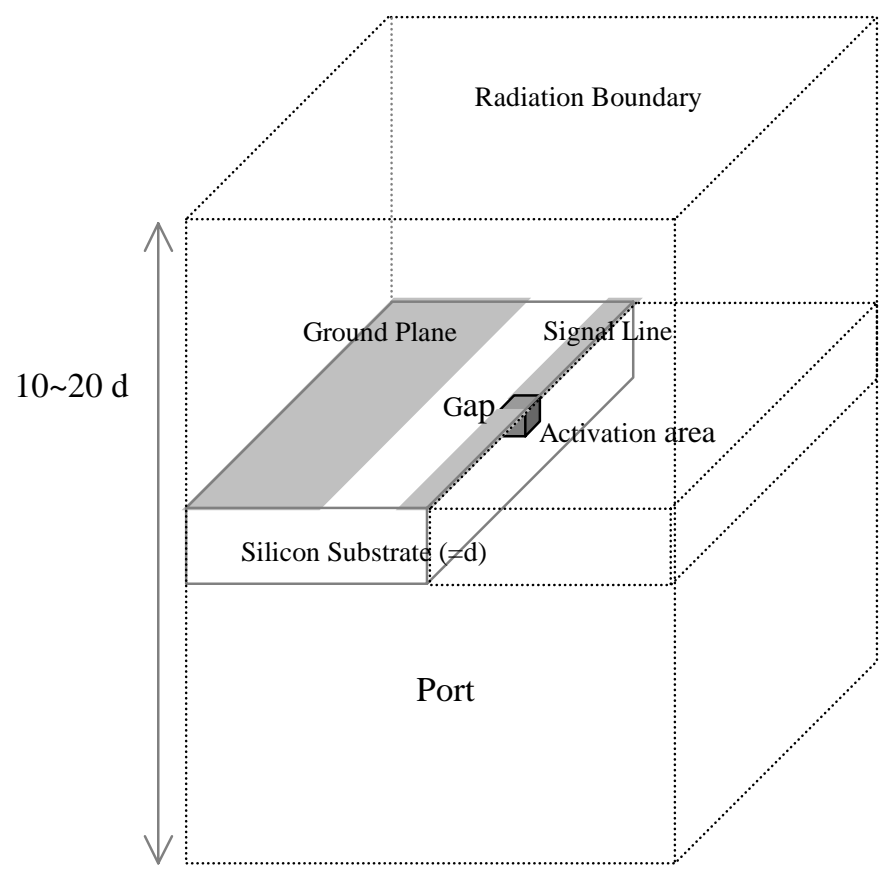

Fig. 5. A model setup for the HFSS simulation of the CW-mode OMS based on a silicon coplanar waveguide. 
Transmission lines such as coplanar waveguide and microstrip lines can be solved using the HFSS, and the scattering parameters are obtained as a result. Fig. 5 shows the basic model setup for the HFSS simulation of the CWmode OMS based on a coplanar waveguide. All the dimensions are the same as we used for the experiments. The dimensions used for the model are: substrate thickness is $350 \mu \mathrm{m}$, ground plane width is $800 \mu \mathrm{m}$, signal line width is 120 $\mu \mathrm{m}$, and the space between the signal line and ground plane is $80 \mu \mathrm{m}$. However, we have applied the shorter signal line, which is reduced to $1000 \mu \mathrm{m}$. Thus, the required memory and the running time could be reduced. The general rule for the boundary setup of the CPW is that the horizontal radiation boundaries are the same as the total length from one ground plane to the other ground plane, and the vertical radiation boundaries used are approximately 10 times the substrate thickness. Then, we need to set the boundary filled with the air, and the boundary plane is assigned with the radiation. The ground planes and the signal lines are assigned with the two-dimensional perfect conductor surfaces. The traditional ports are used. Thus, two-dimensional cross-sections of the front and back side radiation boundary are assigned for the ports. To reduce the discretization error of the FEM, it is necessary to refine the finite element mesh locally in the neighborhood of the fine structure area. Both the manual and adaptive meshes are applied.

Assuming that there is no carrier diffusion, $5 \times 10^{6} \mathrm{~S} / \mathrm{m}$ of $\Delta \sigma_{o}$ (surface photoconductivity with considering the carrier recombination) is obtained with $880 \mathrm{~mW} / \mathrm{mm}^{2}$ of the incident optical power density, which corresponds to 10 $\mathrm{mW}$ optical power with a $120 \mu \mathrm{m}$ beam diameter. This calculation shows that the gap region becomes a good conductor with the optical power. Other quantities used for the calculation are: $\alpha=1 \times 10^{4} \mathrm{~cm}^{-1}, \tau=10^{-4}$ sec, $S=0.5$, and $R=0.3$. The surface photoconductivity is exponentially decreased from the surface of the substrate to the bottom. However, we assume that the effective photoconductivity is the same as $\Delta \sigma_{o}$, and the effective plasma depth is the same as $1 / \alpha$ as defined in Platte's paper ${ }^{12}$. As we expect, close to $0 \mathrm{~dB}$ of insertion loss has been obtained with $5 \times 10^{6} \mathrm{~S} / \mathrm{m}$ of the effective photoconductivity and $1 \mu \mathrm{m}$ of the effective plasma depth from the HFSS simulations as shown in Fig. 7 (a).

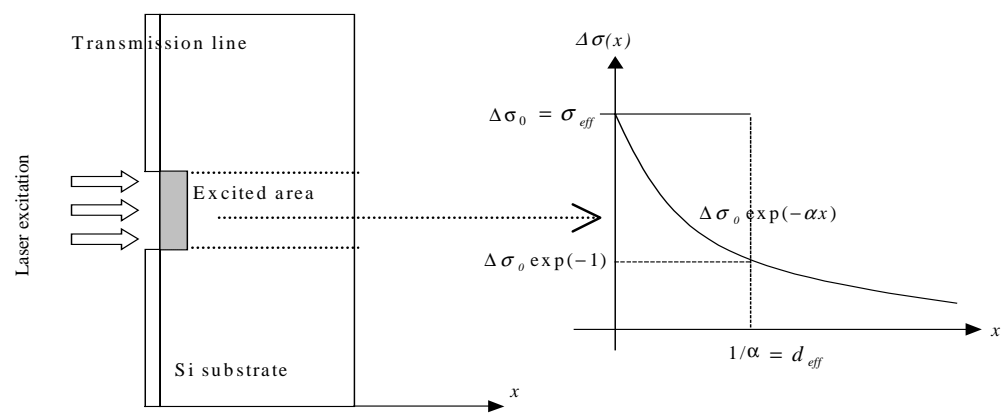

(a)

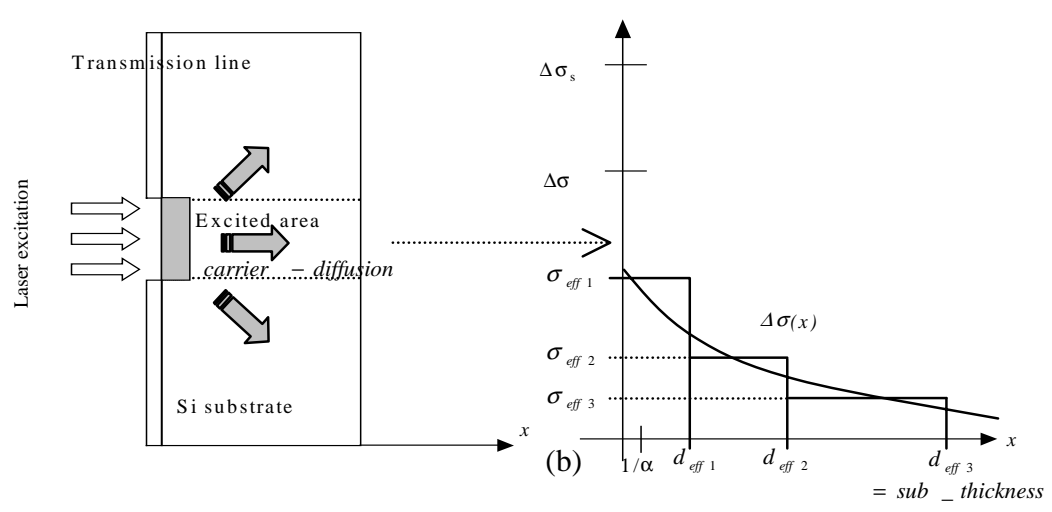

Fig. 6. HFSS simulation model with the effective photoconductivity and the effective plasma depth: (a) without considering carrier diffusion (b) with considering carrier diffusion. 


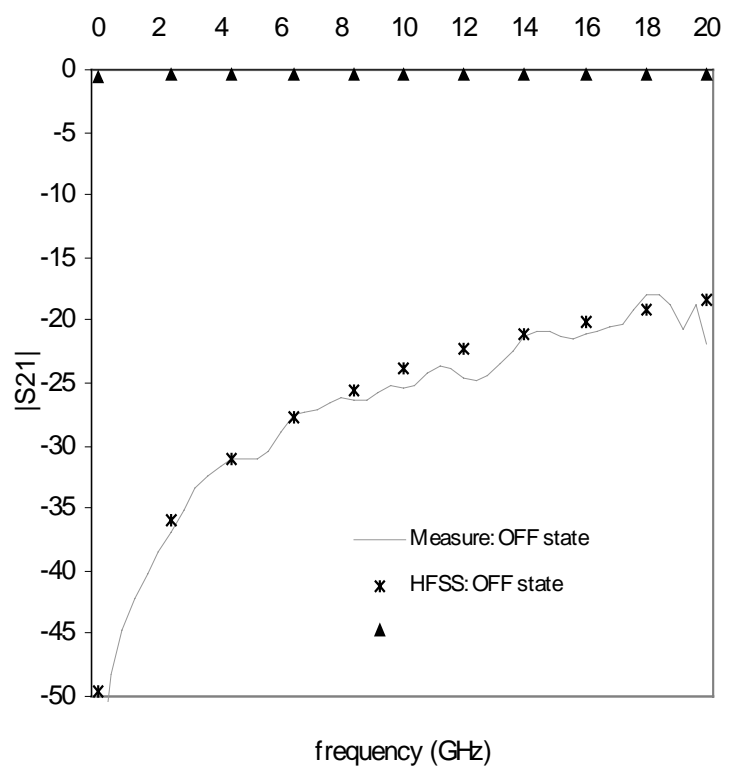

(a)

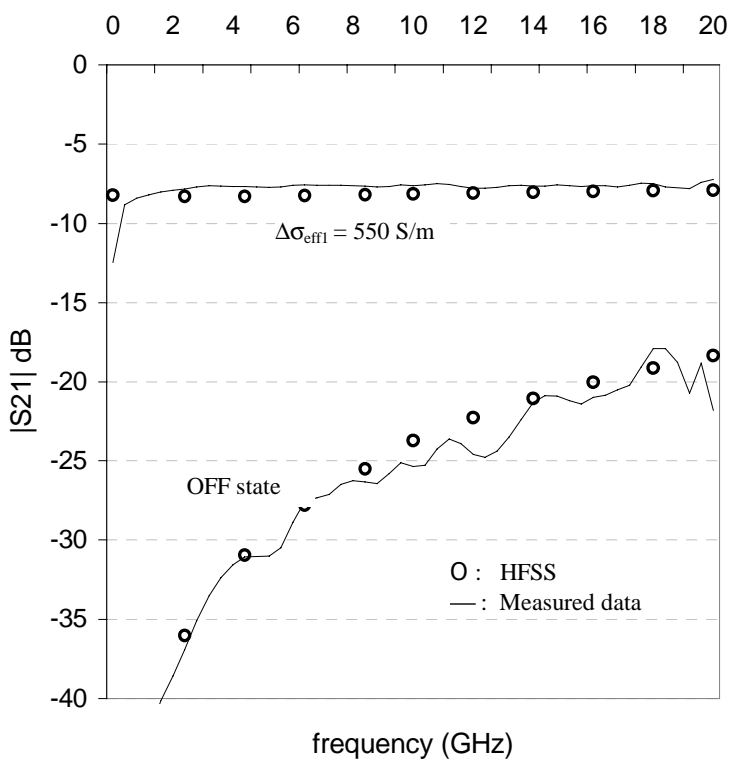

(b)

Fig. 7. (a) HFSS simulation results for the case without considering carrier diffusion (b) Measurement for the best-case insertion loss and the HFSS simulations with respect to the effective photoconductivity.

However, we have measured $8 \mathrm{~dB}$ of the best-case insertion loss with the same incident optical power density $\left(880 \mathrm{~mW} / \mathrm{mm}^{2}\right)$. We know that the main reason for the difference between the measurement and the simulation is due to carrier diffusion in the CW-mode operation. Based on this experiment, it has been found that carrier diffusion is one of the main reasons of the loss in the $\mathrm{CW}$-mode operation. The effective photoconductivity and the effective plasma depth in CW-mode operation are well defined and derived in Platte's paper ${ }^{12}$. Using the analytic forms, we may be able to estimate the effective photoconductivity of the gap region and the effective plasma depth. However, as the diffusion length of the carriers is getting longer with the longer carrier lifetime, errors due to the uniformly defined photoconductivity over the plasma depth are increased. Another way to obtain the effective photoconductivity is an inverse method using the measured insertion loss and the numerical simulation results. To estimate the photoconductivity more correctly, the gap area is separated into three regions. Those are effective plasma depths of 1,2 , and 3 from the top of the substrate surface to the bottom. Each region has different effective photoconductivity as shown in Fig. 6 (b). Fig. 7 shows both the measured insertion loss with $10 \mathrm{~mW}$ of the incident optical power and the simulated insertion loss with different effective photoconductivity. $\Delta \sigma_{e f f 2}$ and $\Delta \sigma_{e f f 3}$ are assigned with $80 \%$ of the upper layer's effective photoconductivity. Thus, if the $\Delta \sigma_{\text {eff } 1}$ is obtained, $\Delta \sigma_{\text {eff } 2}$ and the $\Delta \sigma_{\text {eff } 3}$ can be decided by a simple calculation. As shown in Fig. 7 (b), $8 \mathrm{~dB}$ of insertion loss corresponds to approximately $550 \mathrm{~S} / \mathrm{m}$ of $\Delta \sigma_{\text {eff } 1}$, which is approximately $0.01 \%$ of the $\Delta \sigma_{o}$. As a result, only $0.01 \%$ of the generated carriers contribute to the photoconductivity.

\section{CONCLUSIONS}

A detailed analysis for the CW-mode OMS has been performed. Contrary to the picosecond photoconductivity, adverse influences are observed in $\mathrm{CW}$-mode operation mainly due to the carrier recombination and carrier diffusion. And hence, it may not be possible to obtain enough free carriers for close to $0 \mathrm{~dB}$ of insertion loss. Therefore, we have shown that a semiconductor material having a long carrier lifetime can be applicable for the substrate of the CW-mode OMS. Also we have provided the reason that a microstrip line can be used only under very limited conditions in CWmode operation, and hence, the CPW may be the proper structure. In a microstrip line, the plasma behaves as a lossy media rather than a conducting channel. A simulation model for the gap-structure OMS has been set, and the insertion loss have been obtained from the calculated effective photoconductivity. We also defined the effective photoconductivity for three different plasma layers, and the effective photoconductivity of each layer can be inversely obtained from the 
measured data. Finally, we have found that only $0.01 \%$ of the generated carriers contribute to the photoconductivity if the carrier diffusion is involved, and it may not be possible to obtain close to $0 \mathrm{~dB}$ of insertion loss without proper carrier confinement structure.

\section{ACKNOWLEDGEMENTS}

This work is supported by the National Science Foundation (ECS-9908849).

\section{REFERENCES}

1. C. H. Lee, "Picosecond optics and microwave technology, " IEEE Trans. MTT-38 (5), pp. 596-607, May 1990.

2. A. M. Johnson and D. H. Auston, "Microwave switching by picosecond photoconductivity, " IEEE J. of Quantum Electronics, QE-11 (6), pp. 283-287, June 1975.

3. V. A. Manasson, L. S. sadovnik, V. A. Yepishin, and D. Marker, "An optically controlled MMW beam-steering antenna based on a novel architecture," IEEE Trans. MTT-45 (8), pp. 1497-1500, August 1997.

4. R. C. Dempsey, and R. M. Bevensee, "The synaptic antenna for reconfigurable array applications," 1989 IEEE APS International Symposium, San Jose, CA, 1989.

5. J. L. Freeman, B. J. Lamberty, and G. S. Andrews, "Optoelectronically reconfigurable monopole antenna," Electronics letters, 28 (16), pp. 1502-1503, July 1992.

6. C. K. Sun, R. Nguyen, C. T. Chang, and D. J. Albares, "Photovoltaic-FET for optoelectronic RF/microwave switching," IEEE Trans. MTT-44 (10), pp. 1747-1750, October 1996.

7. W. C. Yih, Microwave photoconductivity investigations in silicon and in polymers, MS Thesis, University of Washington, 1997.

8. S. Lee, U. Ketprom and Y. Kuga, "Optically controlled switches on a coplanar waveguide," 2000 National Radio Science Meeting/URSI, January 8 11, 2001 Boulder, CO.

9. S. Lee, S. Lee, U. Ketprom and Y. Kuga, "Optically CW-mode controlled microwave switches with carrierconfinement on a coplanar waveguide," 2001 IEEE AP-S International Symposium, July 8-13, 2001, Boston, MA.

10. M. A. Omar, Elementary Solid State Physics, Addison-Wesley Publishing Company, Inc. 1975.

11. W. Platte, "Optoelectronic microwave switching, " IEE Proceedings, vol. 132, Pt. J, no. 2, pp. 126-132, April 1985.

12. W. Platte, "Effective photoconductivity and plasma depth in optically quasi-CW controlled microwave switching devices," IEE Proceedings, vol. 135, Pt. J, no. 3, June 1988.

13. W. Platte and B. Sauerer, "Optically CW-induced losses in semiconductor coplanar waveguides," IEEE Trans. MTT- 37 (1), pp. 139-149, January 1989. 